\title{
3D PEEC Capacitance Calculations
}

\author{
Jonas Ekman \\ Luleå University of Technology \\ Luleå, Sweden \\ Jonas.Ekman@sm.luth.se
}

\author{
Giulio Antonini, Antonio Orlandi \\ University of L'Aquila \\ Poggio di Roio, Italy
}

\begin{abstract}
The partial element equivalent circuit ( $P E E C$ ) method has shown to be useful in mixed circuit and electromagnetic analysis. In PEECs, the extensions from two to three dimensional modelling are mainly in the calculation of the partial self and mutual capacitive couplings. The considerable increase in problem size for $3 D$ PEEC models result in a large number of partial elements that has to be calculated. This results in excessive calculation times if the capacitive calculation routines are poorly constructed. In this paper it is shown that by using local reduction matrices for the capacitive calculations, the calculation time for PEEC model capacitance matrices can be decreased while keeping the accuracy.
\end{abstract}

\section{Keywords}

PEEC, equivalent circuits, capacitance calculations.

\section{INTRODUCTION}

In the PEEC method [1, 2], conductors and dielectrics are represented by partial circuit elements. The partial elements for simple PEEC models are lumped inductances, capacitances and resistances that are calculated based on a discretization of a structure. For PEEC models including retardation the capacitive and inductive couplings must be delayed by the free space travel time between the source and field point disabling the use of most commercial circuit simulation software like SPICE [3]. Instead the circuit equations can be directly formulated and solved using a suitable method, for example, the modified nodal analysis (MNA) approach [4]. The notation $\left(L_{p}, P, R, \tau\right)$ PEEC are used for PEEC models to emphasize the inclusion of each group of partial elements where $\left(L_{p}\right)$ is the partial inductances, $(P)$ the partial coefficients of potential, $(R)$ the losses in conductors and dielectrics and $(\tau)$ the retarded electric and magnetic field couplings.

Two discretizations are performed in the PEEC method, first the capacitive or surface cell discretization accounting for the charge distribution and electric field coupling and then the inductive or volume cell discretization representing the current flow in the structure and the magnetic field couplings. The discretization level and thus the number of partial elements are determined by conductors shapes and separations and most important the upper frequency limit of the PEEC model. A rule of thumb is to use 20 cells per wavelength. When a geometry allows a discretization into orthogonal surface or volume cells the partial elements can be calculated using fast closed-form expressions. For non-orthogonal geometries [5], numerical integration routines must be used.

For PEEC models several surface cells can be connected to the same node or connection point, comparable to a SPICE circuit node. The routine to reduce and combine the coefficients of potential of multiple surface cells to one single node coefficient of potential [6] is influencing the partial element calculating time and accuracy of the resulting PEEC model. This paper proposes three novel solutions for the reduction from surface- to node- coefficients of potential for the PEEC method. In the last section resulting node coefficients of potentials for the different methods are compared for basic PEEC model building blocks and a realistic PEEC model example is also given.

\section{THE PEEC METHOD}

The theoretical derivation for the PEEC method starts from the expression of the electric field, at field point $\vec{r}$, by using the scalar and vector potentials $\phi$ and $\vec{A}$ respectively.

$$
\vec{E}(\vec{r})=-j \omega \mu \vec{A}(\vec{r} \prime)-\nabla \phi(\vec{r} \prime)
$$

where $\vec{r}$ denotes a source point. If the field point, $\vec{r}$, is on the surface of a conductor and the definitions of the potentials are used with the free space Green's function, $G\left(\vec{r}, \vec{r}^{\prime}\right)$, Eq. (1) can be written as

$$
\begin{aligned}
\vec{E}^{i}(\vec{r}) & =\frac{\vec{J}(\vec{r})}{\sigma}+j \omega \mu \int_{v^{\prime}} G(\vec{r}, \vec{r} \prime) \vec{J}\left(\vec{r}^{\prime}\right) d v \prime \\
& +\frac{\nabla}{\varepsilon_{0}} \int_{v^{\prime}} G\left(\vec{r}^{\prime}, \vec{r}^{\prime}\right) q(\vec{r} \prime) d v \prime
\end{aligned}
$$

where $\vec{E}^{i}$ is an external applied electric field and $\vec{J}$ is the current density and $\sigma$ is the conductivity at the field point, at the conductor. This integral equation and the 


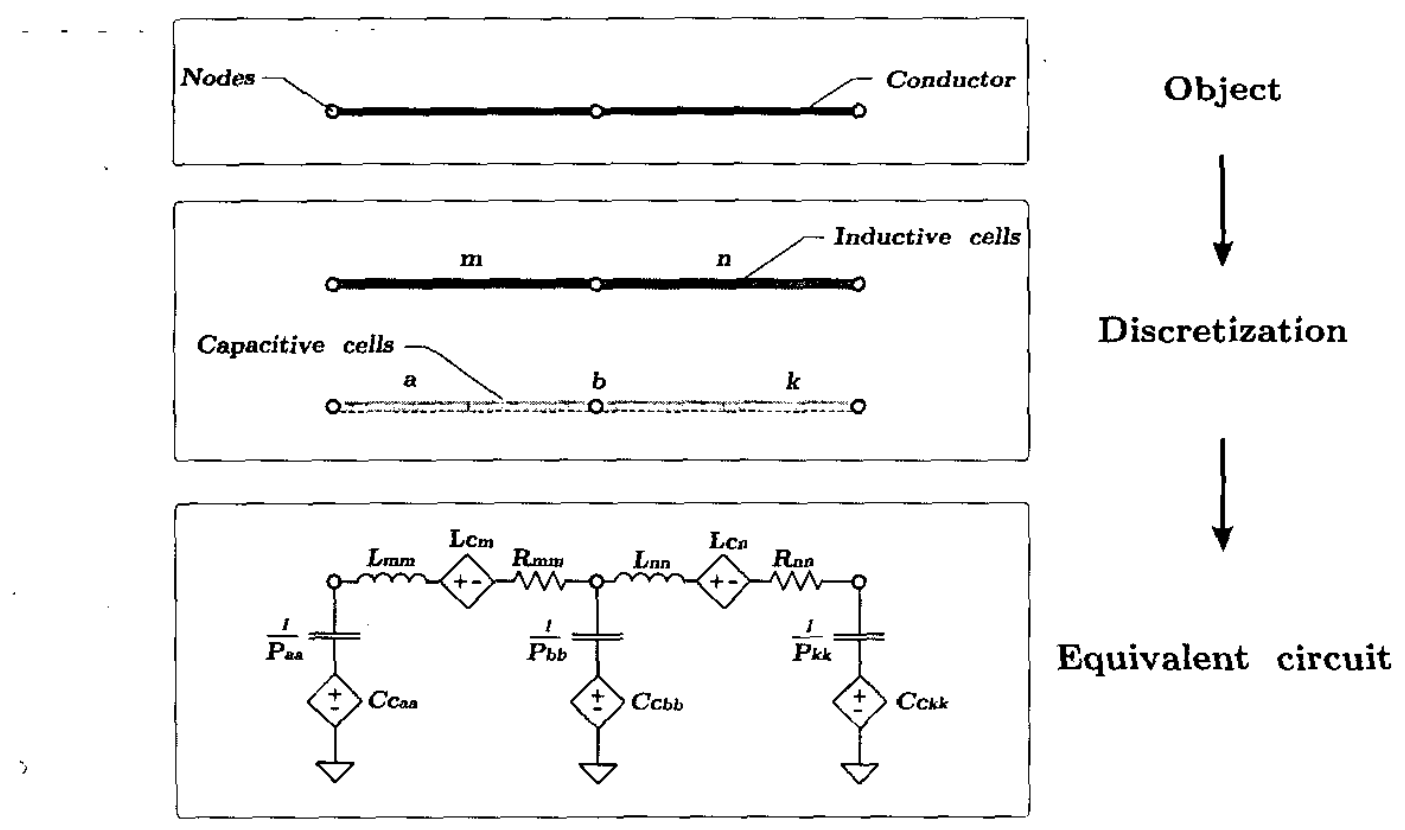

Figure 1: PEEC discretization and equivalent circuit example.

concept of partial elements are the base of the PEEC method. For a more complete theoretical derivation see for example [7].

If the PEEC method is applied to a perfect conductor, Object in Fig. 1, and two inductive cells are used in the length direction, entitled $m$ and $n$ in the figure. Then the corresponding PEEC two cell model consists of two partial self inductances, entitled $L_{m m}$ and $L_{n n}$, and two volume cell DC resistances, $R_{m m}$ and $R_{n n}$ between the nodes. The capacitive coupling is realized by one partial self pseudo-capacitance [8] to each node, $C_{a}=\frac{1}{P_{a a}}, C_{b}=\frac{1}{P_{b b}}$ and $C_{k}=\frac{1}{P_{k k}}$. To account for the retarded electric and magnetic field couplings between the capacitive and inductive partitions, controlled. voltage sources are used in the example in the figure, $C_{\mathrm{c}}$ and $L_{c}$. As can be noted from Fig. 1, the capacitive cells (light grey) are shifted with regard to the inductive cells (dark grey) by half a cell length. This is due to a central difference approximation in the theoretical derivation [7].

The advantages with the PEEC method is noted in the simple example in Fig. 1. First, the method transforms the electromagnetic field problem to a circuit representation and offers a combined solution making it easy to include additional circuit elements such as transmission lines and power sources. Second, the same model can be used for time- and frequency-domain modelling. Third, the method offers a great deal of flexibility in the calculation of the partial elements (accuracy and speed) and macro-modelling techniques can be used to simplify large problems.

\section{REDUCTION FROM SURFACE TO PEEC NODE COEFFICIENTS OF POTENTIAL}

For one and two dimensional PEEC models multiple surfaces can be associated to the same PEEC node if two discretized objects are put together. This is exemplified in the $1 D$ PEEC example in Fig. 2 where the two shaded capacitive cells share the same node. In three dimensional PEEC models, using a edge based formulation, up to three surfaces can share the same node in one object. This is also depicted in Fig. 2 where the three shaded surfaces of the 3D PEEC geometry share the same node, indicated with black circles. For each PEEC object a surface coefficients of potential matrix, $\mathbf{P}_{S}$, can be written. For the 1D PEEC example in Fig. 2 the $\mathbf{P}_{S}$ matrix is seen in Eq. (3) where $p_{s i j},(1 \leq i \leq 4)$ and $(1 \leq j \leq 4)$, are the coefficients of potential for the different surfaces.

Since the surfaces share the same PEEC node a combined self coefficient of potential need to be calculated from Eq. (3) to enable the derivation of the circuit equations. Also, combined mutual coefficients of potentials have to be calculated taking to account all surfaces in the PEEC's.

$$
\mathbf{P}_{S}=\left[\begin{array}{llll}
p_{s 11} & p_{s 12} & p_{s 13} & p_{s 14} \\
p_{s 21} & p_{s 22} & p_{s 23} & p_{s 24} \\
p_{s 31} & p_{s 32} & p_{s 33} & p_{s 34} \\
p_{s 41} & p_{s 42} & p_{s 43} & p_{s 44}
\end{array}\right]
$$

The following sections displays three different techniques for the reduction from surface to PEEC node 
coefficients of potentials. The accuracy is enforced by theoretical conditions and node coefficients of potential are displayed and compared for basic PEEC building block geometries and for a simple application.
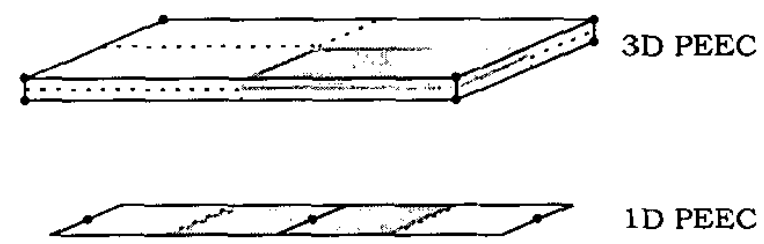

Figure 2: Examples showing situations when multiple surfaces share the same node.

\section{Global reduction approach, $\mathbf{R}_{G}$}

The first technique is considered to be the most accurate since the theoretical conditions enforces:

1. An equi-potential condition to the node points in the complete PEEC model, meaning that the node potential before and after reduction is the same.

2. The charge conservation law to the complete PEEC model. This condition enforces the summation of charges for the surface connected to the same node.

This is done by introducing a reduction matrix, $\mathbf{R}_{G}$, that operates on the global surface coefficients of potential matrix, $\mathbf{P}_{S}$ to create the reduced node coefficients of potential matrix $\mathbf{P}_{N}$. In the global $\mathbf{P}_{S}$ the capacitive interactions between all the surfaces are displayed, consider Eq. (3) for the 1D structure in Fig. 2, while in the $\mathbf{P}_{N}$ all the capacitive interactions between the nodes are displayed.

The $\mathbf{R}_{G}$ matrix is constructed by writing out the node voltages and total charges before and after reduction as

$$
\begin{aligned}
& \mathbf{V}_{N}=\mathbf{R}_{G} \mathbf{V}_{S} \\
& \mathbf{Q}_{S}=\mathbf{R}_{Q} \mathbf{Q}_{N}
\end{aligned}
$$

where subscript $S$ and $N$ represents PEEC model variables before (surface representation) and after reduction (node representation). In $\mathrm{Eq}_{\mathrm{q}}$. (5) $\mathbf{R}_{Q}$ is the charge reduction matrix created as $\mathbf{R}_{G}$. By writing the node voltages and total charges in this way the two equations, Eq. (4) and Eq. (5), can be linked by the relationship $\mathbf{V}=\mathbf{P} \mathbf{Q}$ to form $\mathrm{Eq}$. (6).

$$
\mathbf{Q}_{N}=\mathbf{R}_{Q} \mathbf{P}_{S}^{-1} \mathbf{R}_{G} \mathbf{V}_{N}
$$

The conditions enforced by the global reduction approach results in $\mathbf{R}_{Q}=\mathbf{R}_{G}^{T}$ and the node coefficients of potential matrix can be written as Eq. (7)

$$
\mathbf{P}_{N}=\left(\mathbf{R}_{G}^{T} \mathbf{P}_{S}^{-1} \mathbf{R}_{G}\right)^{-1}
$$

Consider the 1D PEEC model in Fig. 2 where the second and third surfaces (shaded) share the same node. The reduction matrix is then written as Eq. (8)

$$
\mathbf{R}_{G}=\left[\begin{array}{lll}
1 & 0 & 0 \\
0 & 1 & 0 \\
0 & 1 & 0 \\
0 & 0 & 1
\end{array}\right]
$$

And the surface coefficients of potential matrix is reduced from a $4 \times 4$ to the $3 \times 3$ node coefficients of potential matrix.

The drawback of this method are the matrix multiplications and inversions in Eq. (7) that has to be performed on large dense matrices, $\mathbf{P}_{N}\left[\mathrm{n}_{n} \times \mathrm{n}_{n}\right]$ and $\mathbf{P}_{S}\left[\mathrm{n}_{s} \times \mathrm{n}_{s}\right]$ where $\mathrm{n}_{n}$ and $\mathrm{n}_{s}$ are the number of nodes and surfaces respectively and $n_{n} \leq n_{8}$. The size of the $\mathbf{P}_{S}$ matrix can, in the worst case, be three times the size of the $\mathbf{P}_{N}$ matrix. For example, consider the $3 \mathrm{D}$ structure in Fig. 2 where the object is discretized into $\left(n_{s}=\right) 24$ surface cells (dashed lines) and $\left(n_{n}=\right) 8$ corresponding nodes (solid black circles).

The accuracy for this approach is guaranteed by the theoretical conditions enforced in the derivation of the reduction matrix, $\mathbf{R}_{G}$. Therefore, this method will be considered as the reference in the comparisons in Section Numerical Experiments.

\section{Local reduction approach, $\mathbf{R}_{L}$}

The matrix operations on the large matrices in Eq. (7) are avoided in the second and third method where the node coefficients of potential matrix, $\mathbf{P}_{N}$, is built without creating the surface coefficients of potential matrix, $\mathbf{P}_{S}$, and the corresponding matrix operations.

In the second method the same conditions as for the Global reduction approach are enforced for the actual node/nodes involved in the reduction while the rest of the system is not considered. The advantage is that the size of the $\mathbf{P}_{S}$ matrix is equal to the number of surfaces involved in the reduction for each node. The reduction matrix, $\mathbf{R}_{L}$, is constructed in the same way as before but for the nodes involved in the actual computation. The $\mathbf{P}_{N}$ matrix for the 1D PEEC model in. Fig. 2 is then calculated according to Eq. (9) - (12) where the $p_{s i j}$ elements are the same as in Eq. (3).

$$
\mathbf{P}_{N}=\left[\begin{array}{ccc}
p_{s 11} & \mathbf{A}(1,2) & p_{s 14} \\
\mathbf{A}(2,1) & \mathbf{C}(1) & \mathbf{B}(1,2) \\
p_{s 41} & \mathbf{B}(1,2) & p_{s 44}
\end{array}\right]
$$

$$
\begin{gathered}
\mathbf{A}= \\
{\left[\begin{array}{lll}
1 & 0 & 0 \\
0 & 1 & 1
\end{array}\right]\left[\begin{array}{lll}
p_{s 11} & p_{s 12} & p_{s 13} \\
p_{s 21} & p_{s 22} & p_{s 23} \\
p_{s 31} & p_{s 32} & p_{s 33}
\end{array}\right]\left[\begin{array}{ll}
1 & 0 \\
0 & 1 \\
0 & 1
\end{array}\right]}
\end{gathered}
$$




$$
\begin{gathered}
\mathbf{B}= \\
{\left[\begin{array}{lll}
1 & 1 & 0 \\
0 & 0 & 1
\end{array}\right]\left[\begin{array}{lll}
p_{s 22} & p_{s 23} & p_{s 24} \\
p_{s 32} & p_{s 33} & p_{s 34} \\
p_{s 42} & p_{s 43} & p_{s 44}
\end{array}\right]\left[\begin{array}{ll}
1 & 0 \\
1 & 0 \\
0 & 1
\end{array}\right]} \\
\mathbf{C}=\left[\begin{array}{ll}
1 & 1
\end{array}\right]\left[\begin{array}{ll}
p_{s 22} & p_{s 23} \\
p_{s 32} & p_{s 33}
\end{array}\right]\left[\begin{array}{l}
1 \\
1
\end{array}\right]
\end{gathered}
$$

Since the 1D PEEC object in Fig. 2 is a small system the speed up using this Local reduction approach is negligible. But, for larger systems the speed up is substantial, see Section Numerical Experiments.

The Local reduction approach enforces the equipotential and conservation of charges to the local system. Therefore the method decouples the local system and performs the reduction from surface to node coefficients of potentials. The error introduced by the reduction is hard to quantify for a general PEEC model. But, the impact on single node coefficients of potential for basic PEEC geometries and a simple example is displayed in Section Numerical Experiments.

Local weighting approach, $R_{W}$

The third method uses a weighting mean value procedure to calculate the node coefficients of potential matrix, $\mathbf{P}_{N}$. The calculation is based on the areas, $A_{i}$, $0<i \leq n_{s}$, and the surface coefficients of potentials, $p_{s_{i j}}$, for the capacitive surface patches, as shown in Eq. (13).

$$
p_{n_{i j}}=\frac{\sum_{i} \sum_{j} p_{s_{i j}}\left(A_{i}+A_{j}\right)}{A_{T O T}}
$$

For equal surface cell areas, $A_{i}=A_{j}$, this corresponds to the arithmetic mean value. In Eq. (13) the node coefficients of potential $\mathbf{P}_{n}$ is calculated without any matrix operations and is therefore the fastest method.

The equi-potential condition is fulfilled as for the other approaches. But the conservation of charges condition is transformed to depend on the charge distribution that in turn is considered in the surface coefficients of potential calculations, ie the orientation and position of the surface cells.

\section{CIRCUIT EQUATIONS FOR PEEC PROBLEMS}

It is important to notice the use of the node coefficients of potential matrix, $\mathbf{P}_{N}$, when forming the circuit equations for the final PEEC model. When using the modified nodal approach the $\mathbf{P}_{N}$-matrix is used directly while for other formulations such as nodal (admittance matrix) formulations the inverse of the $\mathbf{P}_{N}$-matrix is used. This can result in the preferred use of the global reduction approach for the latter formulations since the accuracy is higher and one matrix inversion has to be done, the one in Eq. (7) or the one to transform the $\mathbf{P}_{N^{-}}$

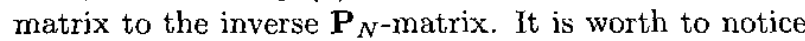
the wide use of the modified nodal approach for automatic formulation of circuit equations in circuit analysis software and also for PEEC based EM solvers.

\section{NUMERICAL EXPERIMENTS}

The three methods have been implemented in a PEEC based full-wave solver. The numerical experiments presented in the following subsections involve computation of the self-and mutual-node coefficients of potential for basic PEEC geometries/PEEC model building blocks. A parallel plate capacitor is used to exemplify the impact of the different calculation techniques on PEEC model results.

\section{Node coefficients of potential calculations}

This section displays the impact of the different calculation routines on self- and mutual-node coefficients of potential calculations. The results are displayed in Fig. 3 to 7 with a node coefficient of potential relative error, $P_{\text {rel.err. }}$, defined as in Eq. (14).

$$
P_{\text {rel.err. }}=\frac{P_{i j}-P_{i j_{R_{G}}}}{P_{i j_{R_{G}}}}
$$

In Eq. (14) $P_{i j}$ is the calculated node coefficient of potential (node-cop), using the local reduction- or weighting-approach, and $P_{i j_{H_{G}}}$ is the same node-cop calculated using the global reduction approach.

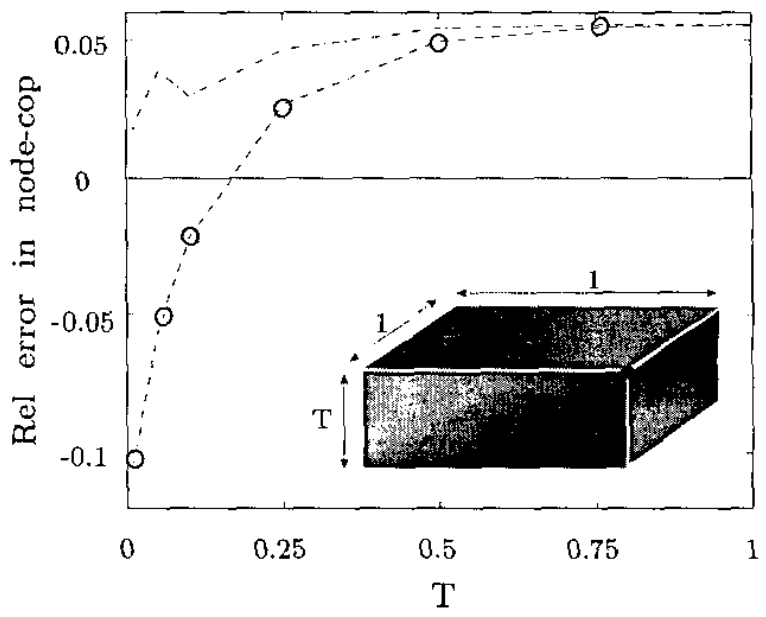

Figure 3: Three surfaces to one node reduction. Relative error for the node-cop. $R_{G}$ (solid), $R_{L}$ (dash-dot), $R_{W}$ (dash-circled)

In the results the global reduction approach is considered as the reference, thus the zero relative error: indicated in the figures. All the figures show a reduced node-cop. relative error, Eq. (14), for nodes made up of homogenous patches when using the local weighting approach. The error is predominantly larger using the local weighting approach than for the local reduction approach. The maximum relative error using $R_{L}$ is 0.07 , while the maximum error using $R_{W}$ is 0.13 . It can be noted that the relative error is larger for the calculation of the self node-cop. compared to the mutual term when using the local reduction approach. 
Table 1: Capacitance relative error and $\mathbf{P}_{N}$ calculation speed up using different reduction approaches

\begin{tabular}{|c|c|c|c|c|c|c|c|c|}
\hline \multicolumn{2}{|c|}{ Ind. disc. } & \multicolumn{2}{|c|}{ Cap. cells } & \multicolumn{5}{|c|}{ Cap. reduction method \& results } \\
\hline \multirow[t]{2}{*}{ L \& W } & \multirow[t]{2}{*}{$\mathrm{T}$} & \multirow[t]{2}{*}{$\mathrm{n}_{s}$} & \multirow[t]{2}{*}{$\mathrm{n}_{n}$} & $\overline{\mathbf{R}_{G}}$ & \multicolumn{2}{|c|}{$\mathbf{R}_{L}$} & \multicolumn{2}{|c|}{$R_{W}$} \\
\hline & & & & Cerror & $C_{\text {error }}$ & Speed up & $C_{\text {error }}$ & Speed $u p$ \\
\hline 1 & 0 & 32 & 16 & 0.025 & 0.150 & 1 & 0.150 & 1 \\
\hline 5 & 1 & 360 & 210 & 0.026 & 0.123 & 22 & 0.022 & 29 \\
\hline 1 & 1 & 72 & 24 & 0.025 & 0.012 & 1 & 0.092 & 1 \\
\hline 5 & 0 & 192 & 144 & 0.028 & 0.153 & 16 & 0.151 & 19 \\
\hline
\end{tabular}

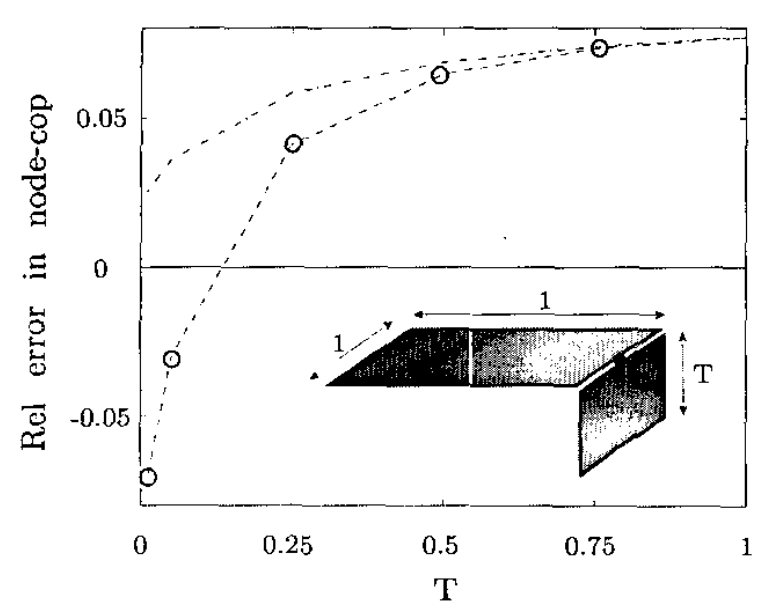

Figure 4: Two surfaces to one node reduction. Relative error for the node-cop. $R_{G}$ (solid), $R_{L}$ (dash-dot), $R_{W}$ (dash-circled).

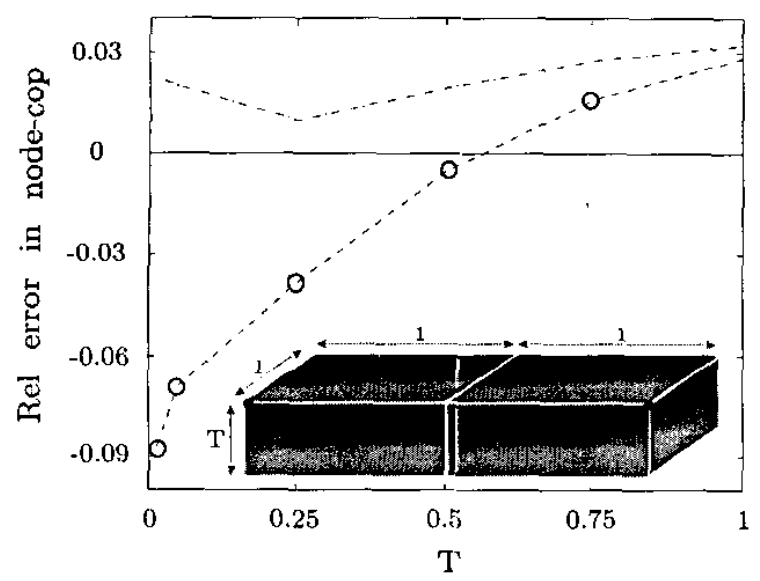

Figure 5: Six surfaces to two nodes reduction. Relative error for the node-cop. $R_{G}$ (solid), $R_{L}$ (dash-dot), $R_{W}$ (dash-circled).

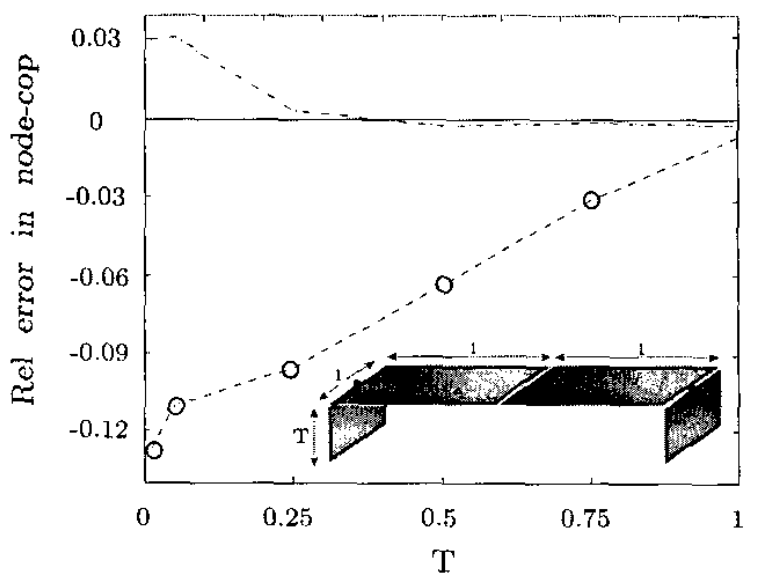

Figure 6: Four surfaces to two nodes reduction. Relative error for the node-cop. $R_{G}$ (solid), $R_{L}$ (dash-dot), $R_{W}$ (dash-circled).

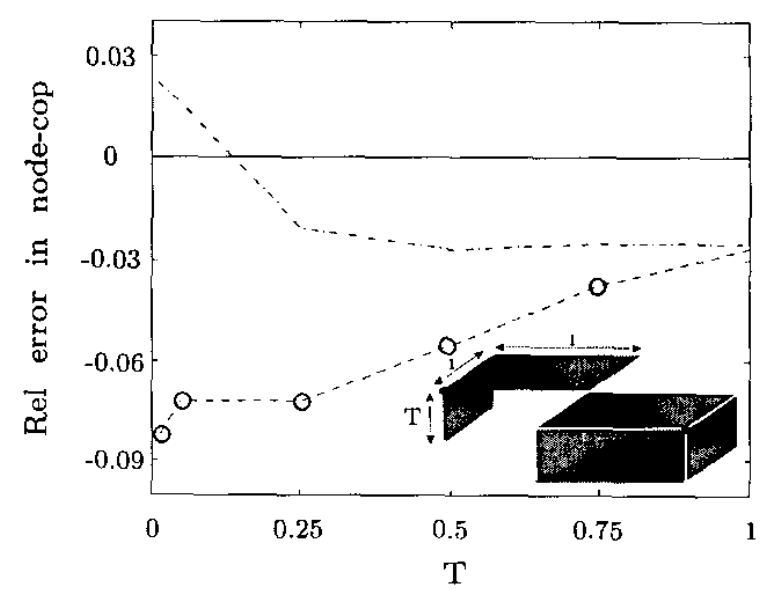

Figure 7: Five surfaces to two nodes reduction. Relative error for the node-cop. $R_{G}$ (solid), $R_{L}$ (dash-dot), $R_{W}$ (dash-circled). 


\section{Parallel plate capacitor}

To display the impact of the different node-cop calculation routines a $10 \times 10 \mathrm{~cm}(\mathrm{~L} \times \mathrm{W})$ parallel plate capacitor is modelled. The capacitor geometry is chosen since the capacitive effects are important in the resulting PEEC model and thus displays the impact of the different calculation routines effectively. The capacitor plates are $50 \mu \mathrm{m}$ thick (T) and spaced $1 \mathrm{~mm}$ apart by a dielectric material with relative permittivity of 3.4 resulting in a theoretical capacitance value of $0.3011 \mathrm{nF}$. The capacitance value is then calculated or modelled based on $\left(L_{p}, P, R, \tau\right)$ PEEC frequency domain simulations while varying.

- The 3D capacitance reduction methods:

$$
\begin{aligned}
& -\mathbf{R}_{G} . \\
& -\mathbf{R}_{L} . \\
& -R_{W} .
\end{aligned}
$$

- The capacitor discretization:

-1 or 5 inductive cells for $\mathrm{L}$ and $\mathrm{W}$ directions for both conducting plates and dielectric material.

- Zero thickness or 1 inductive cell for the conductor plate thicknesses $(\mathrm{T})$.

The results from the test are shown in Table 1 where the Speed up is defined as $\frac{T_{R_{G}}}{T}$, where $T_{R_{G}}$ is the calculation time for the $\mathbf{P}_{N}$ matrix using the Global reduction approach and $T$ is the current $\mathbf{P}_{N}$ matrix calculation time. The $C_{\text {error }}$ is a relative error defined as

$$
C_{\text {error }}=\frac{\left|C-C_{A}\right|}{C_{A}}
$$

where $C_{A}$ is the analytical capacitance value, $0.3011 \mathrm{nF}$, and $C$ is the capacitance value from simulations.

The impact on the modelled capacitance value for the different formulations is substantial, see Table 1, with a maximum relative error of $15 \%$. The $\left(L_{p}, P, R, \tau\right)$ PEEC simulations based on the Global reduction approach is predicting the capacitance value best with a maximum relative error of $2.8 \%$. The other formulations displays a variation in relative error but also a considerable speed up, in the order of 22 times, for this small problem.

\section{CONCLUSIONS}

Three different formulations for the calculation of the PEEC model node coefficients of potential is presented and evaluated. The global reduction approach offer theoretically very good accuracy but suffers from the matrix operations involved resulting in large computation times. The local reduction approach performs the same matrix operations on maximum two nodes at a time thus reducing the computational effort and a considerable speed up is obtained. The third method calculates the node-cop by using a local weighting procedure for the individual surfaces but suffers from poor accuracy for certain cell aspect ratios. To be noted is that the speed up is strongly dependent on the speed and efficiency of the matrix routines used in the PEEC implementation. However the speed up is increasing proportional to problem size, ie number of nodes and surfaces in the PEEC model, when using the local reduction approach or the local weighting approach instead of the global reduction approach. For the parallel plate capacitor example, consisting of maximum 210 nodes, the speed up is in the order of 22 times with a maximum relative error of $15 \%$. For one test, in Table 1, the local weighting approach showed a reduced relative error and a speed up of 29 times compared to the global reduction approach.

\section{REFERENCES}

[1] A. E. Ruehli. Inductance Calculations in a Complex Integrated Circuit Environment. IBM Journal of Research and Development, 16(5):470-481, September 1972.

[2] A. E. Ruehli and P. A. Brennan. Efficient Capacitance Calculations for Three-Dimensional Multiconductor Systems. IEEE Transactions on microwave Theory and Techniques, 21(2):76-82, February 1973.

[3] L. W. Nagel. SPICE: A computer program to simulate semiconductor circuits. Elecrt. Res. Lab. Report ERL M520, University of California, Berkeley, May 1975.

[4] C. Ho, A. Ruehli and P. Brennan. The Modified Nodal Approach to Network Analysis. IEEE Transactions on Circuits and Systems, pages 504-509, June 1975 .

[5] A. E. Ruehli et al. Non-Orthogonal PEEC Formulation For Time And Frequency Domain Modeling. To be published in: IEEE Transactions on EMC, Special issue on "Advanced EMC Numerical Modeling", May 2003.

[6] A. E. Ruehli, P. A. Brennan and H. W. Young. Recent Progress in Capacitance Computation Methods. In: Proc. of the IEEE Int. Symp. on Circuits and Systems, Phoenix, Az, USA, 1975.

[7] J. E. Garrett. Advancements of the Partial Element Equivalent Circuit Formulation. Ph.D. Dissertation, The University of Kentucky, 1997.

[8] H. Heeb and A. E. Ruehli. Approximate TimeDomain Models of Three-Dimensional Interconnects. In: Proc. of the IEEE Int. Conference on Computer Design, pages 201-205, 1990. 